University of Nebraska - Lincoln

DigitalCommons@University of Nebraska - Lincoln

Faculty Publications from the Department of Engineering Mechanics

February 2008

\title{
Influence of spatial correlation function on attenuation of ultrasonic waves in two-phase materials
}

Dalie Liu

University of Nebraska - Lincoln

Joseph A. Turner

University of Nebraska - Lincoln, jaturner@unl.edu

Follow this and additional works at: https://digitalcommons.unl.edu/engineeringmechanicsfacpub

Part of the Mechanical Engineering Commons

Liu, Dalie and Turner, Joseph A., "Influence of spatial correlation function on attenuation of ultrasonic waves in two-phase materials" (2008). Faculty Publications from the Department of Engineering Mechanics. 11.

https://digitalcommons.unl.edu/engineeringmechanicsfacpub/11

This Article is brought to you for free and open access by the Mechanical \& Materials Engineering, Department of at DigitalCommons@University of Nebraska - Lincoln. It has been accepted for inclusion in Faculty Publications from the Department of Engineering Mechanics by an authorized administrator of DigitalCommons@University of Nebraska - Lincoln. 


\title{
Influence of spatial correlation function on attenuation of ultrasonic waves in two-phase materials
}

\author{
Dalie Liu and Joseph A. Turner ${ }^{a)}$ \\ Department of Engineering Mechanics, University of Nebraska-Lincoln, W317.4 Nebraska Hall, Lincoln, \\ Nebraska 68588, USA
}

(Received 28 June 2007; revised 15 January 2008; accepted 15 February 2008)

\begin{abstract}
Successful processing of materials by powder sintering relies on the creation of strong interparticle bonds. During certain critical stages of the sintering process, the medium may be modeled as two phases consisting of the particles and a surrounding matrix. Ultrasonic methods have been proposed as a potential tool for monitoring such sintering processes. Thus, an understanding of the propagation and scattering of elastic waves in two-phase solids is of fundamental importance to these monitoring techniques. In this article, expressions for the ultrasonic attenuations are developed based on the spatial statistics of the density and Lamé parameters of the material constituents under assumptions of statistical homogeneity and statistical isotropy. The formulation is based on the solution of the elastodynamic Dyson equation within the limits of the first-order smoothing approximation. Since the geometric two-point correlation function plays a central role in the model, numerical models are developed using Voronoi polycrystals surrounded by a matrix of different material properties. The spatial statistics of the medium are extracted from these models. The results presented suggest new ultrasonic techniques may be developed to extract multiple correlation lengths for such two-phase microstructures. (c) 2008 Acoustical Society of America.
\end{abstract}

[DOI: $10.1121 / 1.2896757$ ]

PACS number(s): 43.35.Cg, 43.20.Bi, 43.20.Gp [PEB]

Pages: $2570-2576$

\section{INTRODUCTION}

Sintered metals and ceramics are very important in modern manufacturing. Many construction and engineering materials are produced by sintering of different crystal powders in mechanical engineering, energetics, metal-working industries, instrument making and electronics. An increased understanding of the sintering process would lead to intelligent process control, greater reliability of the production process, and improved quality of the end products. Therefore, continuous real-time monitoring of the microstructure evolution is necessary. More importantly, such monitoring techniques will provide valuable information for modeling sintering processes. Most current monitoring techniques are not in situ techniques. Ultrasonic and eddy current techniques have great potential for direct measurements during processing. However, eddy current techniques are limited to information predominantly about the material near the specimen surface. ${ }^{1}$ Ultrasonic methods can provide information about the interior microstructure due to the penetration of ultrasonic waves and are capable of inspecting the sintering process at high temperatures. ${ }^{2,3}$ However, the main difficulty with such ultrasonic techniques is the theoretical framework necessary to make the measurements quantitative.

The propagation and scattering of elastic waves through random medium has received considerable attention. ${ }^{4,5}$ The basic premise of these analyses is to treat the material properties as random variables and use statistical methods to analyze the wave propagation. Most of those statistical theories

\footnotetext{
a) Author to whom correspondence should be addressed. Electronic mail: jaturner@unl.edu.
}

require information about the spatial statistics of the microstructure to predict the macroscopic properties of heterogeneous materials. The model of Stanke and Kino ${ }^{6}$ clearly established that the two-point correlation function is important for the attenuation within the second-order perturbation theory. Furthermore, the exponential correlation function used by Stanke and Kino for statistically isotropic, polycrystalline media is widely adopted in many works due to its simplicity. ${ }^{7-11}$ The validity of this geometric autocorrelation function in polycrystalline media was examined by Man et $a l .{ }^{12}$ They found that the exponential formula fails to represent the correlation function for the grain boundary structure of a commercial aluminum alloy and that an alternative function is necessary. Such information will provide valuable insight for modeling the microstructure of complex materials during sintering processes.

In this article, the propagation and scattering of ultrasound in partially sintered materials is examined using a twophase model consisting of the polycrystals and a surrounding matrix. The theory based on a stochastic, elastic wave model presented by Turner and Anugonda ${ }^{10}$ is extended to study the attenuation of elastic waves in two-phase materials. The medium is assumed to be statistically homogeneous and statistically isotropic. The spatial statistics of the microstructure, also known as geometric correlation functions, are numerically evaluated and investigated. The attenuation of longitudinal and transverse elastic wave is presented in terms of the statistics of the density and Lamé parameters of the materials. The influence of correlation functions on the resulting attenuations is discussed. 


\section{STATISTICS OF MATERIAL PROPERTIES}

The derivations for scattering attenuation rely on information regarding the statistics of the microstructure. Explicit expressions for the attenuation of longitudinal and transverse elastic waves used in this article are based upon the general description discussed by Turner and Anugonda. ${ }^{10}$ In that work, the solution for the wave vector in the heterogeneous medium is based on the Dyson equation derived for the elastodynamic case. The Dyson equation was solved within the limits of the first-order smoothing approximation ${ }^{13}$ for which the dimensionless combination of material fluctuation, bare wave number and correlation length must be smaller than unity. For more details, the reader may wish to refer to previous work using this approach. ${ }^{9,10,14}$

The spatial statistics of material properties are presented in this section. The material density, $\rho$, and Lamé parameters, $\lambda$ and $\mu$, are assumed to vary spatially and to have the form

$$
\begin{aligned}
& \rho(\mathbf{x})=\bar{\rho}[1+\delta \rho(\mathbf{x})], \\
& \lambda(\mathbf{x})=\bar{\lambda}[1+\delta \lambda(\mathbf{x})], \\
& \mu(\mathbf{x})=\bar{\mu}[1+\delta \mu(\mathbf{x})],
\end{aligned}
$$

where the over-bar terms are the average quantities and terms with $\delta$ represent a dimensionless measure of the spatial fluctuations about the average. It is assumed that the fluctuations are small and that the material properties are locally isotropic. The second-order statistics of the fluctuations are also required. The covariance of the density is given by

$$
R_{\rho \rho}(\mathbf{x}-\mathbf{y})=\langle\delta \rho(\mathbf{x}) \delta \rho(\mathbf{y})\rangle .
$$

The assumptions of statistical homogeneity and statistical isotropy imply that the correlation functions depend only on the distance, $r$, between the two positions. Thus

$$
R_{\rho \rho}(\mathbf{x}-\mathbf{y})=R_{\rho \rho}(|\mathbf{x}-\mathbf{y}|)=R_{\rho \rho}(r) .
$$

Autocorrelations of Lamé parameters, $R_{\lambda \lambda}$ and $R_{\mu \mu}$, as well as the cross correlation between different parameters $R_{\rho \lambda}$, $R_{\rho \mu}$, and $R_{\lambda \mu}$ can be defined in a similar manner.

For a material consisting of two uniform phases, a general and convenient description of the spatial variation is supplied by introducing the characteristic function ${ }^{15}$

$$
\chi_{1}(\mathbf{x})= \begin{cases}1, & \text { if } x \in \Omega_{1}, \\ 0, & \text { otherwise, }\end{cases}
$$

of the region $\Omega_{1}$ occupied by phase " 1 ." The complement $\Omega_{2}$ is occupied by phase " 2 ," and obviously, its characteristic function is $\chi_{2}(\mathbf{x})=1-\chi_{1}(\mathbf{x})$. Starting from the definition of Eq. (3)

$$
R_{\rho \rho}(r)=\frac{\langle(\rho(\mathbf{x})-\bar{\rho})(\rho(\mathbf{y})-\bar{\rho})\rangle}{\bar{\rho}^{2}} .
$$

The density may then be described in terms of the characteristic functions

$$
\rho(\mathbf{x})=\rho_{1} \chi_{1}(\mathbf{x})+\rho_{2} \chi_{2}(\mathbf{x})
$$

and

$$
\bar{\rho}=\rho_{1}\left\langle\chi_{1}(\mathbf{x})\right\rangle+\rho_{2}\left\langle\chi_{2}(\mathbf{x})\right\rangle,
$$

where $\rho_{1}$ and $\rho_{2}$ are the densities of phase "1" (grains) and "2" (matrix). After substitution into Eq. (5), we have

$$
R_{\rho \rho}(r)=\frac{\left(\rho_{1}-\rho_{2}\right)^{2}\left[\left\langle\chi_{1}(\mathbf{x}) \chi_{1}(\mathbf{y})\right\rangle-\eta_{1}^{2}\right]}{\bar{\rho}^{2}},
$$

where $\eta_{1}=\left\langle\chi_{1}(\mathbf{x})\right\rangle$ is the volume fraction of phase "1." The two-point correlation function $S_{1}=\left\langle\chi_{1}(\mathbf{x}) \chi_{1}(\mathbf{y})\right\rangle$ is noted as the probability that $\mathbf{x}$ and $\mathbf{y}$ are both in phase "1." As a result, the covariance reduces to the two-point moment ${ }^{16} M_{2}$ $=\left\langle\chi_{1}(\mathbf{x}) \chi_{1}(\mathbf{y})\right\rangle-\eta_{1}^{2}$. For any statistically homogeneous medium, this two-point correlation moment, $M_{2}$, should be positive-definite such that its Fourier transform must be positive. ${ }^{16,17}$ Similar derivations apply to the covariance of Lame parameters, $R_{\lambda \lambda}$ and $R_{\mu \mu}$, as well as the cross correlations between different parameters, $R_{\rho \lambda}, R_{\rho \mu}$, and $R_{\lambda \mu}$. by

The spatial Fourier transform of the covariance is given

$$
\tilde{R}(\mathbf{p})=\frac{1}{(2 \pi)^{3}} \int d^{3} r R(\mathbf{r}) e^{i \mathbf{p} \cdot \mathbf{r}},
$$

where $\mathbf{p}$ is the wave vector.

The general expressions of scattering attenuations from Turner and Anugonda ${ }^{10}$ may then be simplified for the case of two-phase materials using the two-point correlation function. In the next section, the two-point correlation function is estimated from numerical simulations. The attenuations are then derived in terms of the two-point correlation function.

\section{CORRELATION FUNCTION}

The numerical models of the two-phase materials are constructed using Voronoi polycrystals surrounded by a matrix. This type of model allows the matrix structure to have different properties from the grain structure as dictated by typical sintering problems. Voronoi polycrystals are widely used in numerical analysis of many engineering problems. These models have been shown to model closely polycrystalline metals, ceramics and microstructures of many materials. ${ }^{18-20}$ In Fig. 1, a Voronoi polycrystal diagram is created using a Voronoi tessellation, ${ }^{21}$ demonstrating the two-phase geometry. A normal distribution of Voronoi nuclei is used such that the scattering theories assume uniform grain size, which is the average diameter $D$ of the grains. The thickness of the matrix $d$ is also assumed uniform. Various combinations of grain size and thickness of interface can be created as needed. Thus, the microstructural and statistical information of the medium may be obtained from such models.

Once such a model is created, the two-point correlation function can be measured in five steps: (i) Choose two random points $\mathbf{x}$ and $\mathbf{y}$ separated by a distance $r$ repeatedly throughout the model; (ii) judge whether the points are inside grains or matrix; (iii) calculate $\delta \rho(\mathbf{x}), \delta \rho(\mathbf{y})$ for each point, and then calculate the covariance $\langle\delta \rho(\mathbf{x}) \delta \rho(\mathbf{y})\rangle$ using the 


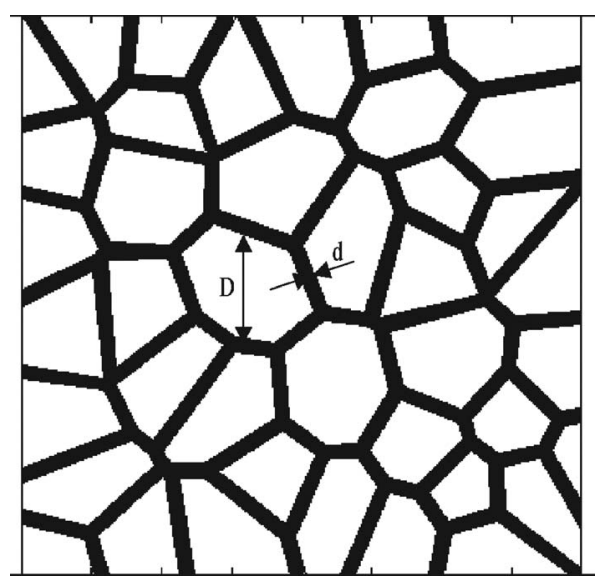

FIG. 1. Two-phase model using Voronoi polycrystals, with the grain diameter (size) $D$ and the thickness of the matrix $d$.

definition of the ensemble average; (iv) average the covariance from different realizations of numerical models (here 100 realizations proved sufficient to obtain converged correlation behavior); (v) deduce the two-point correlation function $S_{1}=\left\langle\chi_{1}(\mathbf{x}) \chi_{1}(\mathbf{y})\right\rangle$ using Eq. (8). Example correlation statistics of density from 100 realizations for a model of size $2.5 \mathrm{~mm}$ by $2.5 \mathrm{~mm}$ by $2.5 \mathrm{~mm}$, grain size $0.8 \mathrm{~mm}$, interface thickness $0.1 \mathrm{~mm}$, are shown in Fig. 2. Note the step size $\Delta r$ used was $0.006 \mathrm{~mm}$ for this result.

Although the two-point correlation function can be acquired directly from numerical simulations, the use of an analytical form is more helpful in evaluation of attenuation. The proper mathematical forms of these functions can allow us to investigate the details of the microstructure and therefore obtain the attenuation of ultrasound in the materials of interest. The use of appropriate forms of the two-point cor-

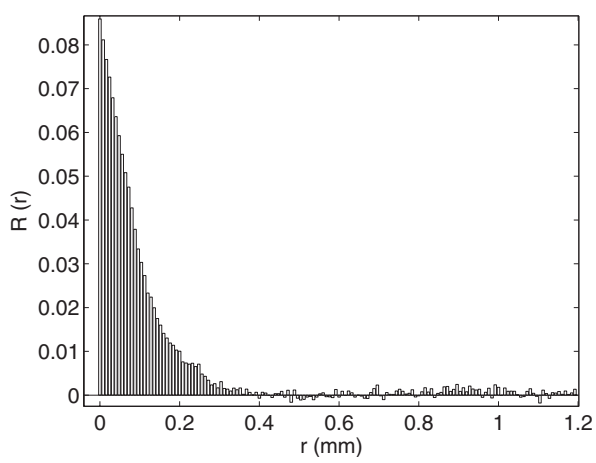

FIG. 2. Histogram of covariance from 100 realizations for a model of size $2.5 \mathrm{~mm}$ by $2.5 \mathrm{~mm}$ by $2.5 \mathrm{~mm}$, grain size $0.8 \mathrm{~mm}$, interface thickness $0.1 \mathrm{~mm}$.

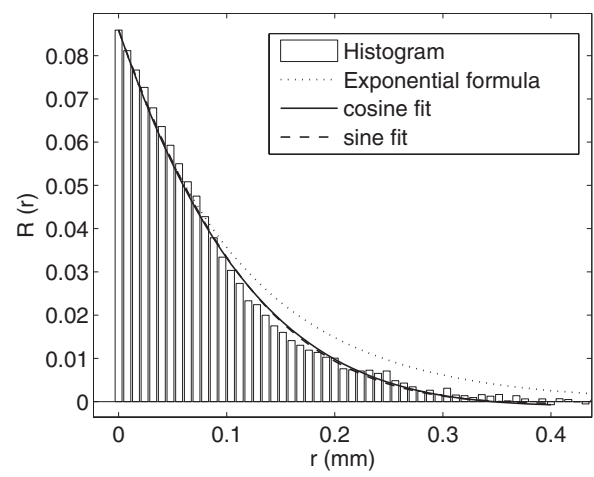

FIG. 3. Least squares fits to the measured data with the exponential formula and other models of correlation functions.

relation functions has been investigated by Torquato and Stell, ${ }^{22-26}$ Corson, ${ }^{27,28}$ and Cule and Torquato, ${ }^{29}$ to name a few. The exponential formula of the correlation function in Stanke and Kino's macroscopically isotropic model has been widely adopted in the studies of ultrasound attenuation in polycrystalline solids. They chose the following spatial autocorrelation function ${ }^{6}$

$$
W(r)=e^{-r / L},
$$

which corresponds to the normalized two-point moment $\left\langle\chi_{1}(\mathbf{x}) \chi_{1}(\mathbf{y})\right\rangle-\eta_{1}^{2}$ in our case. However, as shown in Fig. 3, this exponential formula is not valid to represent the numerical histogram data measured in the two-phase model. As mentioned above, this formula also failed for the geometric autocorrelation function in the grain boundary structure of a commercial aluminum alloy. ${ }^{12}$ Therefore, finding a suitable formula for the two-phase medium is necessary. For a statistically homogeneous, isotropic microstructure, Cule and Torquato, ${ }^{29}$ Yeong and Torquato, ${ }^{30}$ proposed the following two-point correlation functions, respectively:

$$
S_{1}(r)=\eta_{1}^{2}+\eta_{1} \eta_{2} e^{-b r} \frac{\sin (q r)}{q r}
$$

and

$$
S_{1}(r)=\eta_{1}^{2}+\eta_{1} \eta_{2} e^{-b^{\prime} r} \cos (c r),
$$

where $b, q$, and $b^{\prime}, c$ are characteristic spatial scale parameters, and $\eta_{2}=1-\eta_{1}$ is the volume fraction of phase " 2 ." A least squares fit to the measured data using these two correlation functions in Fig. 3 shows that both of them are suitable to represent the two-point correlation function of this twophase model. Note that only the positive part before oscillation is considered for curve fitting due to the short-range order observed. Therefore, the correlation function for the two-phase model is given by

$$
S(r)= \begin{cases}\eta_{1}^{2}+\eta_{1} \eta_{2} e^{\frac{-b r \sin (q r)}{q r}} \text { or } \eta_{1}^{2}+\eta_{1} \eta_{2} e^{-b^{\prime} r} \cos (c r), & 0 \leq r \leq r_{0}, \\ 0, & r>r_{0},\end{cases}
$$




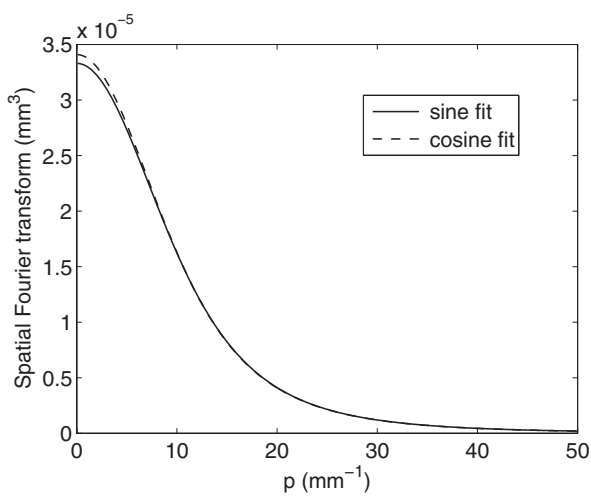

FIG. 4. Comparison of spatial Fourier transforms for the two correlation functions.

where $r_{0}=\pi / q$ or $\pi / 2 c$ for models (11) and (12), respectively. The spatial Fourier transforms of the covariance obtained by curve fitting the histogram for both models are shown in Fig. 4. We found the difference between the two models is very small such that the attenuation results do not vary substantially. It is also found that the characteristic parameters in both models behave very similarly. Therefore, we conclude that both models provide good descriptions of the measured correlation functions. Here, only model (11) is used in the attenuation calculations that follow.

A least squares fit to the histogram of the measured twopoint correlation function provides the two parameters $b, q$ for the model given by Eq. (11). Note that there are also two independent parameters, grain size $D$ and interface thickness $d$, for the two-phase geometry. Next the relationship between $b, q$ and $D, d$ is studied. Given a $2.5 \mathrm{~mm}$ by $2.5 \mathrm{~mm}$ by $2.5 \mathrm{~mm}$ cube, if we have the specified numbers of grains, the sum of $D+d$ is constant even when we change the thickness of the interface $d$. Figure 5 shows how $b$ changes with the thickness $d$ for the cube geometry with 80 grains, where $D$ $+d=0.83$. The characteristic length scale $b^{-1}$ appears to vary linearly with $d$. From Fig. 6, it seems $q$ is a function of $(D$ $+d$ ) because it does not change significantly with $d$. Models with different numbers of grains are also created so that different values of $D+d$ are examined. A similar relationship between $b^{-1}$ and $d$ is observed in Fig. 7 for different combinations of $D+d$. However, from Fig. 8 the physical meaning of $q$ is not clearly related with the geometric parameters, and

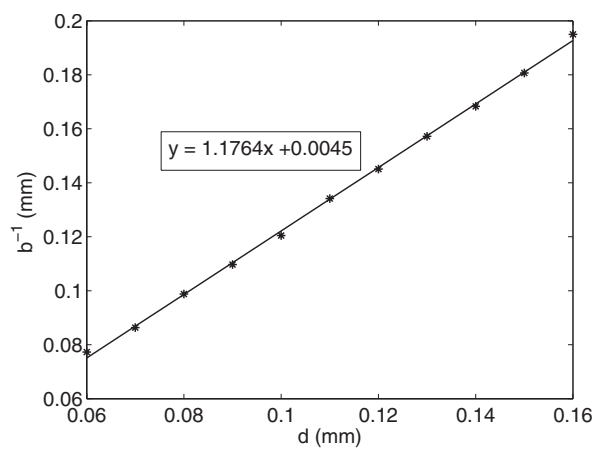

FIG. 5. Characteristic parameter $b$ as a function of $d$ for a $2.5 \mathrm{~mm}$ by $2.5 \mathrm{~mm}$ by $2.5 \mathrm{~mm}$ cubic model with 80 grains.

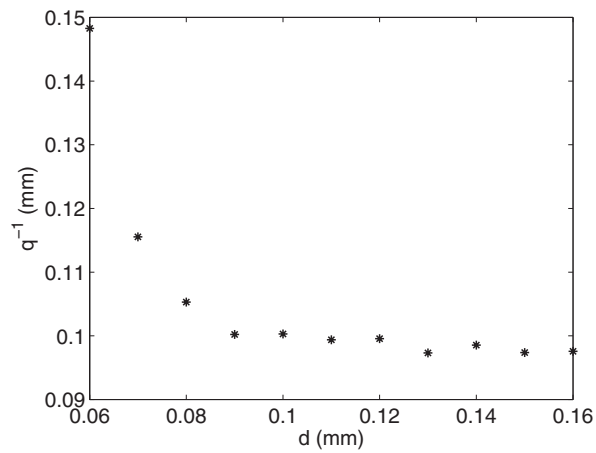

FIG. 6. Characteristic parameter $q^{-1}$ vs. $d$ for one combination of $D+d$ $=0.83 \mathrm{~mm}$.

is still under investigation. We also found out that these two models of correlation functions do not fit well to the histogram when the ratio of $d / D$ is very small. Figure 9 shows the least squares fit to the histogram for the case of $d=0.03, D$ $=0.80$. It is also seen that the exponential formula does not fit the data well. After investigation, it is believed that the ratio $d / D$ needs to be larger than about 0.06 to apply the correlation function of Eq. (11).

The spatial Fourier transform of the two-point moment was then evaluated using numerical integration as

$$
\begin{aligned}
\tilde{S}(\mathbf{p})= & \frac{1}{(2 \pi)^{3}} \int_{0}^{r_{0}} \int_{0}^{\pi} \int_{0}^{2 \pi}\left(\eta_{1} \eta_{2} e^{-b r} \frac{\sin (q r)}{q r}\right) \\
& \times e^{-i p r \cos \theta} \sin \theta r^{2} d r d \theta d \phi .
\end{aligned}
$$

Expressions of attenuation for longitudinal and shear waves can then easily be obtained. In the next section, example numerical results and discussion are presented.

\section{EXAMPLE ATTENUATION RESULTS}

Example results are presented for this two-phase model with known $D$ and $d$. It is first assumed that the material properties are uncorrelated such that the cross correlations are zero $\left(R_{\rho \mu}=R_{\rho \lambda}=R_{\lambda \mu}=0\right)$. In this case, the general expressions of scattering attenuations are given by ${ }^{10}$

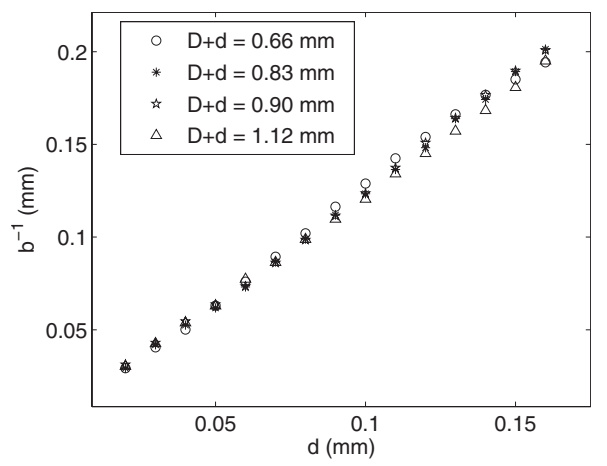

FIG. 7. Characteristic parameter $b$ as a function of $d$ for different combinations of $D+d$.

D. Liu and J. A. Turner: Attenuation in two-phase media

2573 


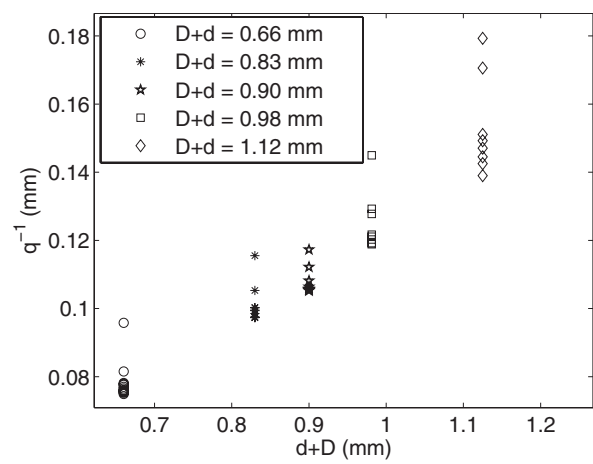

FIG. 8. Characteristic parameter $q^{-1}$ in terms of $D+d$ for different combinations of $D+d$.

$$
\begin{aligned}
\alpha_{L L}= & \frac{\pi^{2} \omega^{4}}{2 c_{L}^{4}} \int_{-1}^{+1}\left\{\chi^{2} \widetilde{R}_{\rho \rho}^{L L}(\chi)+\frac{\left(c_{L}^{2}-2 c_{T}^{2}\right)^{2}}{c_{L}^{4}} \widetilde{R}_{\lambda \lambda}^{L L}(\chi)\right. \\
& \left.+\frac{4 c_{T}^{4}}{c_{L}^{4}} \chi^{4} \widetilde{R}_{\mu \mu}^{L L}(\chi)\right\} d \chi, \\
\alpha_{L T}= & \frac{\pi^{2} \omega^{4}}{2 c_{L} c_{T}^{3}} \int_{-1}^{+1}\left(1-\chi^{2}\right)\left\{\chi^{2} \widetilde{R}_{\rho \rho}^{L T}(\chi)+\frac{\left(c_{L}^{2}-2 c_{T}^{2}\right)^{2}}{c_{L}^{4}} \widetilde{R}_{\lambda \lambda}^{L L}(\chi)\right. \\
& \left.+\frac{4 c_{T}^{2}}{c_{L}^{2}} \chi^{2} \widetilde{R}_{\mu \mu}^{L T}(\chi)\right\} d \chi, \\
\alpha_{T L}= & \frac{1}{2}\left(\frac{c_{T}}{c_{L}}\right)^{2} \alpha_{L T}, \\
\alpha_{T T}= & \frac{\pi^{2} \omega^{4}}{4 c_{T}^{4}} \int_{-1}^{+1}\left\{\left(\chi^{2}+1\right) \widetilde{R}_{\rho \rho}^{T T}(\chi)+\left(1-3 \chi^{2}\right.\right. \\
& \left.\left.+4 \chi^{4}\right) \widetilde{R}_{\mu \mu}^{T T}(\chi)\right\} d \chi,
\end{aligned}
$$

where $\omega$ is the angular frequency. The $\widetilde{R}$ terms are defined by

$$
\widetilde{R}^{i j}=\widetilde{R}\left(\left|\frac{\omega}{c_{i}} \hat{\mathbf{p}}-\frac{\omega}{c_{j}} \hat{\mathbf{s}}\right|\right),
$$

where $i$ and $j$ refer to the wave types, $L$ or $T$ (longitudinal or transverse). Here, $\hat{\mathbf{p}}$ defines the propagation direction and $\hat{\mathbf{s}}$ defines the scattered direction, with $\chi=\hat{\mathbf{p}} \cdot \hat{\mathbf{s}}=\cos \Phi$, where $\Phi$ is the angle between these directions. The correlation func-

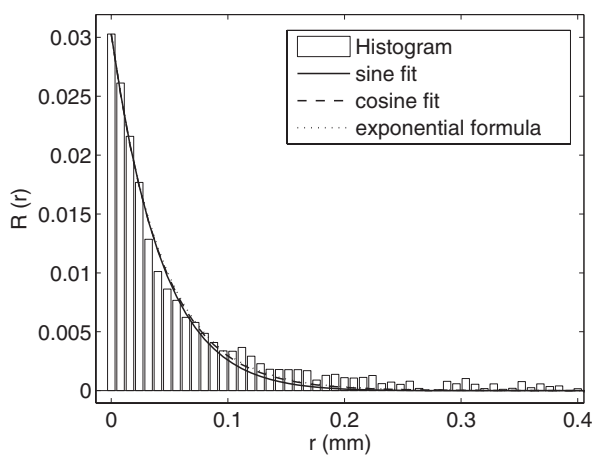

FIG. 9. Fitting of the correlation function for the model with a small ratio of $d / D(d=0.03, D=0.80)$.

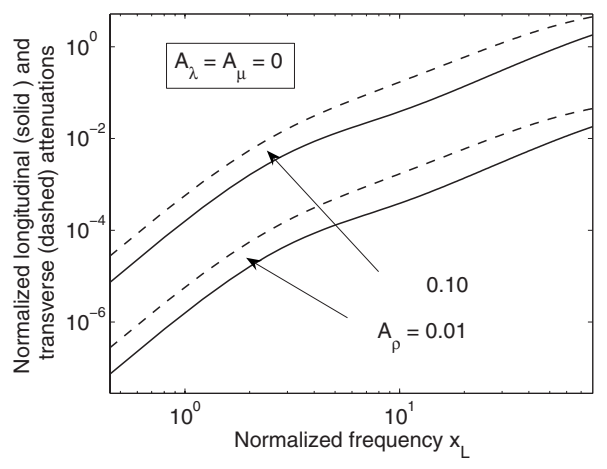

FIG. 10. Normalized longitudinal and transverse attenuations as a function of dimensionless frequency, $x_{L}=\omega D / c_{L}$, for density fluctuations only.

tions depend only on this angle due to the assumption of statistical isotropy. The final expressions for the total attenuations are given by ${ }^{14}$

$$
\begin{aligned}
& \alpha_{L}=\alpha_{L L}+\alpha_{L T}, \\
& \alpha_{T}=\alpha_{T L}+\alpha_{T T} .
\end{aligned}
$$

For the two-phase material, since the material properties are assumed uncorrelated, from Eq. (8) the covariance can be written

$$
R_{\tau \tau}(r)=A_{r}^{2}\left[S(r)-\eta_{1}^{2}\right]
$$

where the subscript $\tau$ refers to the material parameters $\rho, \lambda$ and $\mu$. The contrast of the properties for the two phases is given by $A_{\tau}=\left(\tau_{1}-\tau_{2}\right) / \bar{\tau}$.

For the specific case shown here, the grain size and thickness of the matrix are chosen as $D=0.80 \mathrm{~mm}$ and $d$ $=0.10 \mathrm{~mm}$. The volume fraction of the grains is $\eta_{1}=0.684$. The parameters in the two-point correlation model are $b$ $=8.09 \mathrm{~mm}^{-1}$ and $q=8.90 \mathrm{~mm}^{-1}$. The ratio of wave speed used here is $c_{T} / c_{L}=1 / 2$.

Numerical results of the attenuations are now presented. Figure 10 shows the normalized longitudinal $\alpha_{L} D$ and transverse attenuations $\alpha_{T} D$ as a function of dimensionless frequency, $x_{L}=\omega D / c_{L}$, for density fluctuations only $\left(A_{\lambda}=A_{\mu}\right.$ $=0)$. The attenuations increase with the fourth power of frequency in the low frequency limit as expected. After a transition region, the attenuations scale with the square of frequency. The longitudinal attenuation is always smaller than the transverse attenuation as shown. It can be seen that the contrasts or fluctuations of material properties affect the ratio of the attenuations. Figure 11 shows the normalized longitudinal and transverse attenuations as a function of dimensionless frequency, $x_{L}$, for modulus fluctuations only $\left(A_{p}=0\right)$. Similar results are seen as those in Fig. 10. From Eq. (15), attenuations of all material property fluctuations are just the sum of each component from these figures. Moreover, the contribution of density fluctuations to the attenuation weighs much more than modulus fluctuations for the same level of contrasts. All these results are in qualitative agreement with those of previous work. ${ }^{10}$

Finally, the influence of correlation functions is examined. Figure 12 is a plot of normalized longitudinal attenuations for $A_{\rho}=0.1, A_{\lambda}=A_{\mu}=0$ using the exponential formula 


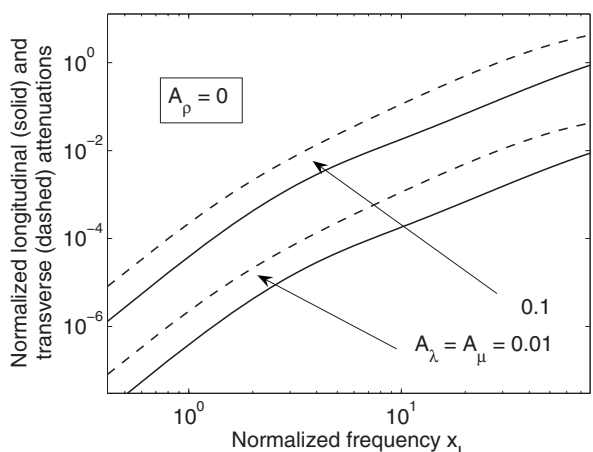

FIG. 11. Normalized longitudinal and transverse attenuations as a function of dimensionless frequency, $x_{L}$, for modulus fluctuations only.

and Cule and Torquato's model, respectively. Attenuations change with the ratio of $b / q$ for this multiple parameter correlation model. When $b / q$ is about 0.25 , the attenuations of two models appear to have overlap in the low frequency range. However, as the frequency increases, the attenuations differ with each other. The results show that the multiple correlation lengths may not be detected at low frequencies. In this specific case, when the dimensionless frequency $x_{L}$ is smaller than about 0.2 , only one correlation length may be extracted. The most likely frequency range to extract both $b$ and $q$ is about $0.3<x_{L}<4$ as observed in this figure. Figure 13 is a plot of normalized transverse attenuations for different correlation functions. Similar results can be observed. The importance of extracting the correlation function from ultrasonic measurements has not been discussed before. All previous attenuation models use correlation functions with a single correlation length. The attenuation results presented here suggest that new ultrasonic techniques for characterization of multiple correlation parameters may be possible. The frequency to be used to extract the multiple correlation parameters may be determined based on the theoretical models developed here. In addition to the analysis presented here, numerical models for attenuation will also be necessary and will be investigated in subsequent work.

\section{SUMMARY}

The propagation of elastic waves in a statistical homogeneous and statistical isotropic two-phase solid has been

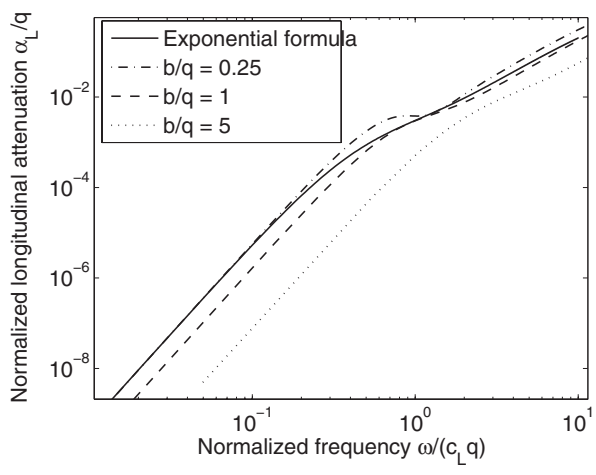

FIG. 12. Comparison of normalized longitudinal attenuations for $A_{\rho}=0.1$, $A_{\lambda}=A_{\mu}=0$ using exponential formula and Cule's correlation function.

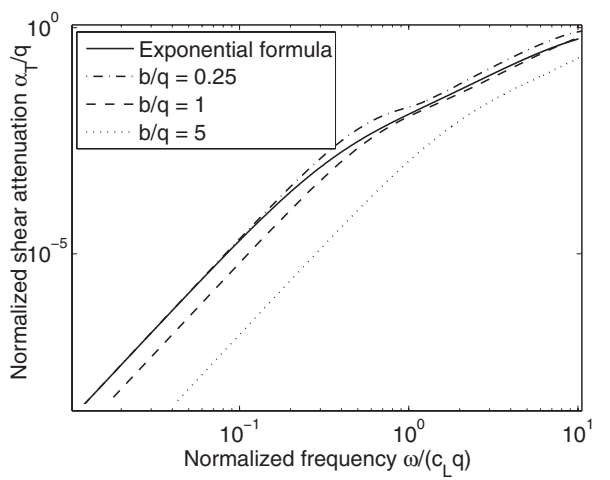

FIG. 13. Comparison of normalized transverse attenuations for $A_{\rho}=0.1$, $A_{\lambda}=A_{\mu}=0$ using exponential formula and Cule's correlation function.

investigated. The theory was derived for the case of both density and Lamé parameters which varied spatially. A numerical method to determine directly the geometric spatial correlation function of the microstructure has been presented. The exponential formula used previously was not applicable for the two-phase model compared with the numerical data. However, the models of two-point correlation functions given by Eqs. (11) and (12) would provide a suitable setting for the two-phase microstructure. The characteristic parameters in these models were also examined. Using those two-point correlation functions, the final expressions of attenuation were given in terms of the spatial statistics of density and Lamé constants. As mentioned, the fluctuations must be small and the volume fraction of each phase must not be so high $(d / D>0.06)$ for the validity of the correlation function model. Influence of the correlation functions on attenuations was observed. The results show that extracting the multiple correlation lengths may only be feasible in a specific frequency range. The general formulation here can be directly related to sintered metals, concrete, and polycrystals with decorated grain boundaries for which previous models had difficulty explaining the experimental results. ${ }^{31,32}$ These results should be very useful for nondestructive testing and materials characterization research despite the simplicity of the assumptions made.

\section{ACKNOWLEDGMENT}

The financial support of the U.S. Department of Energy (Grant No. DE-FG02-01ER45890) is gratefully acknowledged by the authors.

${ }^{1}$ M. J. Johnson and J. R. Bowler, "Pulsed eddy-current measurements for the characterization of thin layers and surface treatments," Review of Progress in Quantitative Nondestructive Evaluation, edited by D. O. Thompson and D. E. Chimenti (Plenum, New York, 1997), Vol. 17A, pp. 251-257.

${ }^{2}$ N. Kulkarni, B. Moudgil, and M. Bhardwaj, "Ultrasonic characterization of green and sintered ceramics: I, time domain," Am. Ceram. Soc. Bull. 73(7), 83-85 (1994).

${ }^{3}$ J. C. Foley and D. K. Rehbein, "In-situ nondestructive evaluation methods for characterizing sintering of aluminum powder metallurgy compacts," P/M Science and Technology Briefs 2(1), 10-13 (2000).

${ }^{4}$ G. C. Papanicolaou, L. V. Ryzhik, and J. B. Keller, "Stability of the P-to-S energy ratio in the diffusive regime," Bull. Seismol. Soc. Am. 86(4), 1107-1115 (1996).

${ }^{5}$ L. V. Ryzhik, G. C. Papanicolaou, and J. B. Keller, "Transport equations 
for elastic and other waves in random media," Wave Motion 24, 327-370 (1996).

${ }^{6}$ F. E. Stanke and G. S. Kino, "A unified theory for elastic wave propagation in polycrystalline materials," J. Acoust. Soc. Am. 75, 665-681 (1984).

${ }^{7}$ F. E. Stanke, "Spatial autocorrelation functions for calculations of effective propagation constants in polycrystalline materials," J. Acoust. Soc. Am. 80, 1479-1485 (1986).

${ }^{8} \mathrm{~S}$. Ahmed and R. B. Thompson, "Propagation of elastic waves in equiaxed stainless-steel polycrystals with aligned [001] axes," J. Acoust. Soc. Am. 99(4), 2086-2096 (1996).

${ }^{9} \mathrm{~J}$. A. Turner, "Elastic wave propagation and scattering in heterogeneous, anisotropic media: Textured polycrystalline materials," J. Acoust. Soc. Am. 106, 541-552 (1999).

${ }^{10} \mathrm{~J}$. A. Turner and P. Anugonda, "Scattering of elastic waves in heterogeneous media with local isotropy," J. Acoust. Soc. Am. 109, 1787-1795 (2001).

${ }^{11}$ L. Yang and J. A. Turner, "Scattering of elastic waves in damaged media," J. Acoust. Soc. Am. 113, 2992-3000 (2003).

${ }^{12}$ C. Man, R. Paroni, Y. Xiang, and E. A. Kenik, "On the geometric autocorrelation function of polycrystalline materials," J. Comput. Appl. Math. 190, 200-210 (2006).

${ }^{13}$ U. Frisch, "Wave propagation in random media," Probabilistic Methods in Applied Mathematics, edited by A. T. Barucha-Reid (Academic, New York, 1968), Vol. 1, pp. 75-198.

${ }^{14}$ R. L. Weaver, "Diffusion of ultrasound in polycrystasls," J. Mech. Phys. Solids 38, 55-86 (1990).

${ }^{15}$ K. Z. Markov and J. R. Willis, "On the two-point correlation function for dispersions of nonoverlapping spheres," Math. Models Meth. Appl. Sci. 8(2), 359-377 (1998).

${ }^{16} \mathrm{~K}$. Z. Markov, "On the correlation functions of two-phase random media and related problems," Proc. R. Soc. London, Ser. A 455, 1049-1066 (1999).

${ }^{17}$ E. Vanmarcke, Random Fields, Analysis and Synthesis (MIT Press, Cambridge, MA, 1983)

${ }^{18}$ S. Ghosh, Z. Nowak, and K. Lee, "Tessellation-based computational methods for the characterization and analysis of heterogeneous microstructures," Compos. Sci. Technol. 57(9), 1187-1210 (1997).

${ }^{19}$ P. D. Zavattieri, P. V. Raghuram, and H. D. Espinosa, "A computational model of ceramic microstructures subjected to multi-axial dynamic loading," J. Mech. Phys. Solids 49(1), 27-68 (2001).
${ }^{20}$ W. Brostow and V. M. Castano, "Voronoi polyhedra as a tool for dealing with spatial structures of amorphous solids and liquids and dense gases," J. Mater. Educ. 21, 297 (1999).

${ }^{21}$ S. Kumar, S. K. Kurtz, J. R. Banavar, and M. G. Sharma, "Properties of a three-dimensional Poisson-Voronoi tesselation: A Monte Carlo study," J. Stat. Phys. 67(3/4), 523-551 (1992).

${ }^{22} \mathrm{~S}$. Torquato and G. Stell, "Microstructure of two-phase random media. I. The N-point probability function," J. Chem. Phys. 77(4), 2071-2077 (1982).

${ }^{23} \mathrm{~S}$. Torquato and G. Stell, "Microstructure of two-phase random media. II. The Mayer-Montroll and Kirkwood-Salsburg Hierarchies," J. Chem. Phys. 78(6), 3262-3272 (1983).

${ }^{24} \mathrm{~S}$. Torquato and G. Stell, "Microstructure of two-phase random media. III. The N-point matrix probability functions for fully penetrable spheres," J. Chem. Phys. 79, 1505-1510 (1983).

${ }^{25} \mathrm{~S}$. Torquato and G. Stell, "Microstructure of two-phase random media. IV. Expected surface area of a dispersion of penetrable spheres and its characteristics function," J. Chem. Phys. 80(2), 878-880 (1984).

${ }^{26} \mathrm{~S}$. Torquato and G. Stell, "Microstructure of two-phase random media. V. The N-point matrix probability functions for impenetrable spheres," J. Chem. Phys. 82, 980-987 (1985).

${ }^{27} \mathrm{P}$. B. Corson, "Correlation functions for predicting properties of heterogeneous materials. I. Experimental measurement of spatial correlation functions in multiphase solids," J. Appl. Phys. 45(7), 3159-3164 (1976).

${ }^{28} \mathrm{P}$. B. Corson, "Correlation functions for predicting properties of heterogeneous materials. II. Empirical construction of spatial correlation functions for two-phase solids," J. Appl. Phys. 45(7), 3165-3170 (1976).

${ }^{29} \mathrm{D}$. Cule and S. Torquato, "Generating random media from limited microstructural information via stochastic optimization," J. Appl. Phys. 86(6), 3428-3437 (1976).

${ }^{30}$ C. L. Y. Yeong and S. Torquato, "Reconstructing random media," Phys. Rev. E 57(1), 495-506 (1998).

${ }^{31}$ X.-G. Zhang, W. A. Simpson, Jr., and J. M. Vitek, "Ultrasonic attenuation due to grain boundary scattering in copper and copper-Aluminum," J. Acoust. Soc. Am. 116(1), 109-116 (2004).

${ }^{32}$ J. Mittleman, K. Y. Han, and R. B. Thompson, "Ultrasonic examination of Ti-6-4 and Ni-trided Ti-6-4 Materials," Review of Progress in Quantitative $N D E$, edited by D. O. Thompson and D. E. Chimenti (Plenum, New York, 1995), Vol. 14, pp. 1457-1464. 